\title{
Desafio Jovem Engenheiro: um torneio para incentivar o ingresso de estudantes de nível médio em carreiras de exatas
}

\author{
Poliana Vitti ${ }^{1}$, Marcos Augusto Francisco Borges ${ }^{1}$ \\ ${ }^{1}$ Faculdade de Tecnologia - Universidade Estadual de Campinas (UNICAMP) \\ Limeira - SP - Brasil \\ p137316@dac.unicamp.br, marcosborges@ft.unicamp.br
}

\begin{abstract}
Brazil has a deficit of 150,000 engineers but data show that Brazilians prefer humanities. Thus, the country needs to arousing of youth's interest about Science, Technology, Engineering and Maths (STEM). Desafio Jovem Engenheiro (DJE) is an online tournament that was proposed to encourage high school students' applications for courses in the STEM fields. The tournament occurs on a project-based learning approach through the proposition of interesting and fun challenges about engineering concepts. In its second edition, DJE involved 326 Brazilian students of four regions of the country, who have engaged and have collaborated to solve each challenge.
\end{abstract}

Resumo. O Brasil apresenta um déficit de milhares de engenheiros e profissionais de Tecnologia da Informação (TI). Dados demonstram que o brasileiro apresenta preferência pelas áreas de humanas, o que justifica o baixo ingresso de jovens em áreas das ciências exatas. O Desafio Jovem Engenheiro (DJE) é um torneio criado para motivar alunos do ensino médio a se candidatarem para cursos na área de exatas, como engenharia e TI. Todo o torneio ocorre através da internet, a partir de projetos desafiadores que usam a abordagem de aprendizagem baseada em projetos. Este trabalho apresenta o DJE, descrevendo sua condução e alguns resultados observados ao longo das duas edições já realizadas.

\section{Introdução}

O Brasil tem enfrentado um período de crescimento econômico pouco expressivo, acarretado, dentre diversos motivos, pela baixa produção de alta tecnologia e pela pouca variedade de produtos de exportação. A exportação brasileira é baseada em commodities que possuem pouco valor agregado. A tecnologia é importada de países como China, Estados Unidos da América (EUA), Alemanha e Japão, que possuem uma grande fatia desse segmento no mercado mundial. Segundo dados do Ministério do Desenvolvimento, Indústria e Comércio Exterior, os bens primários corresponderam a cerca de 50\% das exportações do Brasil em 2014, enquanto os bens de capital e de consumo representaram 66\% das importações [MDIC 2014].

Sabe-se que para um país crescer são necessários investimentos em educação e em pesquisa para gerar novas tecnologias e tornar o mercado mais competitivo [JIN e JIN 2014, KORABLEVA e LITUN 2014]. Para gerar tecnologia de ponta é necessária mão-de-obra especializada [WINTERS 2014]. Entre as principais categorias de profissionais responsáveis pela geração de novas tecnologias estão os engenheiros e 
profissionais da área de Tecnologia da Informação (TI). O Brasil apresenta um déficit de 150 mil engenheiros [CONFEA 2012]. Entretanto o interesse do brasileiro pela área de engenharia e ciências exatas é baixo. De acordo com dados de 2013 do Instituto Nacional de Estudos e Pesquisas Educacionais Anísio Teixeira (INEP) apenas 20\% dos jovens ingressaram em cursos de exatas enquanto $61 \%$ preferiram o campo das ciências humanas. Comparando o Brasil com os países associados à Organização para a Cooperação e Desenvolvimento Econômico ${ }^{1}$ (OCDE), há 17\% de ingressantes a mais nos cursos de Ciências Sociais, Negócios e Direito. Enquanto isso, o ingresso em engenharia, produção e construção é 3\% maior nesses países [INEP 2013].

O objetivo deste artigo é apresentar a aplicação de uma abordagem de aprendizagem baseada em projetos que tem como finalidade incentivar o ingresso de jovens em carreiras de exatas. Essa aplicação se deu por meio de um torneio educacional chamado Desafio Jovem Engenheiro (DJE), que envolveu estudantes do ensino médio de diversas regiões do Brasil.

Este documento está dividido em sete seções, incluindo esta. A Seção 2 discute a falta de profissionais de exatas no Brasil e a baixa procura por cursos de Engenharia. A Seção 3 apresenta trabalhos correlatos a partir de exemplos de iniciativas semelhantes ao DJE. A Seção 4 é dedicada à apresentação do DJE como ferramenta para atrair jovens para cursos de exatas. A Seção 5 descreve os materiais e métodos. A Seção 6 é destinada a apresentar os resultados e dados do torneio. A Seção 7 apresenta as considerações finais.

\section{A Falta de profissionais de exatas no Brasil}

A área das ciências exatas tem baixa procura pelos estudantes brasileiros. Por conta de dificuldades encontradas nas disciplinas de matemática, física e química no ensino básico, muitos estudantes estabelecem uma espécie de bloqueio para aprender e dominar este conteúdo [RASK 2010]. Uma consequência desse bloqueio diz respeito ao crescente desinteresse pelos jovens por programação e pela área de Tecnologia da Informação (TI). Além disso, esse bloqueio acaba por tornar as humanidades mais atrativas do que os cursos de exatas, o que demanda estratégias para torná-los mais interessantes [MENESES et. al 2015].

A Figura 1 é baseada no Censo da Educação Superior de 2013 elaborado pelo INEP sobre o ingresso de alunos em cursos de graduação a cada 10.000 habitantes. Nota-se que o ingresso de estudantes é maior em cursos cuja área está envolvida com as ciências humanas, por exemplo, Ciências Sociais, Negócios, Direito e Educação. A Figura 2 apresenta uma comparação com os países associados à OCDE, no qual se pode observar que o Brasil obteve $17 \%$ de ingressantes a mais nas áreas de Ciências Sociais, Negócios e Direito. Além disso, o interesse pela área da Educação é 15\% maior no Brasil, enquanto o ingresso em engenharia, produção e construção é 3\% menor em relação aos países membros da OCDE.

\footnotetext{
${ }^{1}$ Organisation for Economic Co-operation and Development (OECD) é uma organização mundial sediada na França que conta com 34 países associados, cuja missão é promover políticas que melhorem o bemestar econômico e social de pessoas do mundo todo ${ }^{[\mathrm{OECD} 2016]}$.
} 
Há também alta evasão de alunos nos cursos de engenharia. Nos EUA, entre os anos de 2003 e 2009, 69\% abandonaram seus cursos até 2009 [CHEN e SOLDNER 2013]. Na Figura 3, é possível perceber que o cenário brasileiro é ainda pior. No ano de $2013,80 \%$ dos ingressantes abandonaram seus cursos de engenharia e aproximadamente $70 \%$ abandonaram seus cursos nas áreas de ciências, matemática e computação, as taxas de abandono mais altas entre todos os cursos.

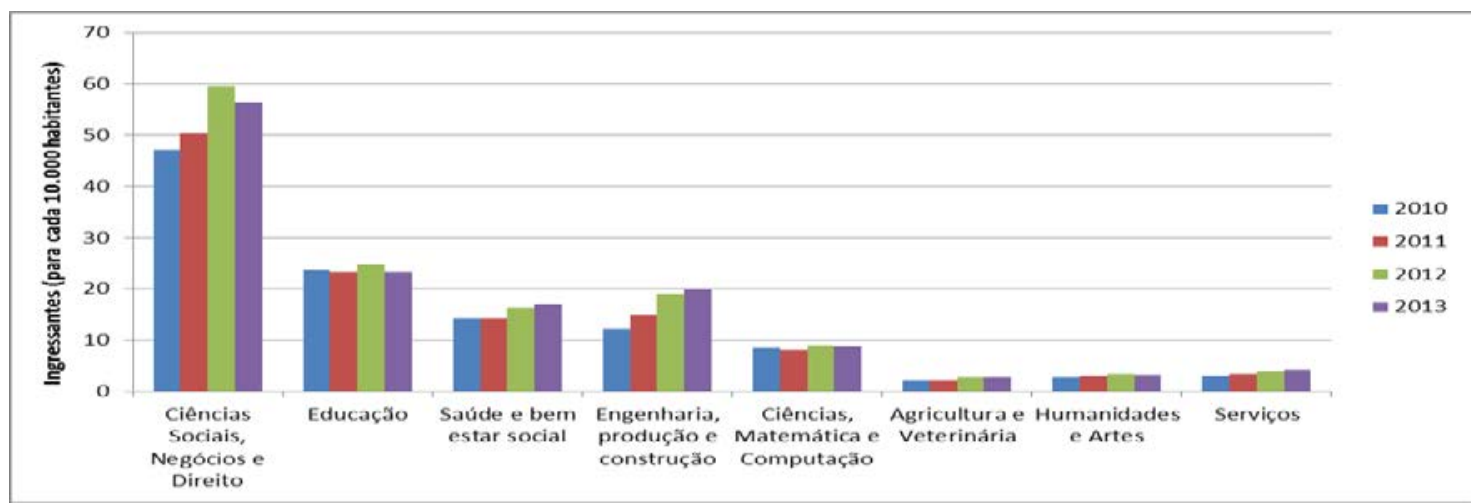

Figura 1. Número de Ingressos em Cursos de Graduação no Brasil para cada 10 mil habitantes, segundo Área Geral do Curso.

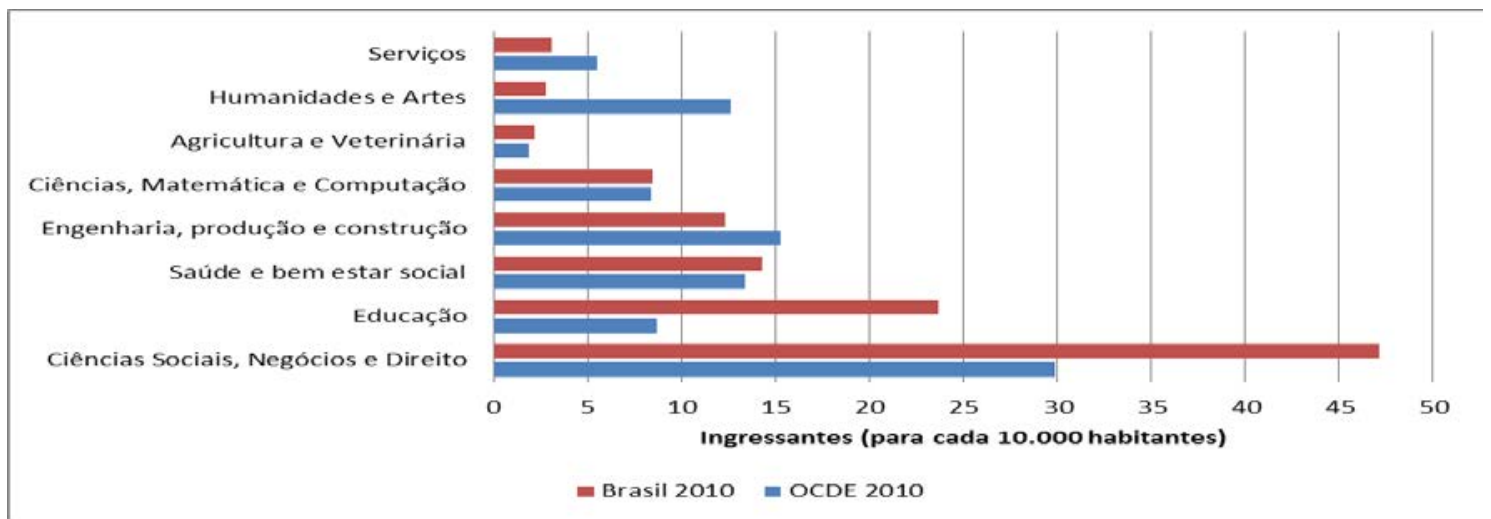

Figura 2. Comparação de ingressantes em cursos superiores entre o Brasil e a OCDE em 2010.

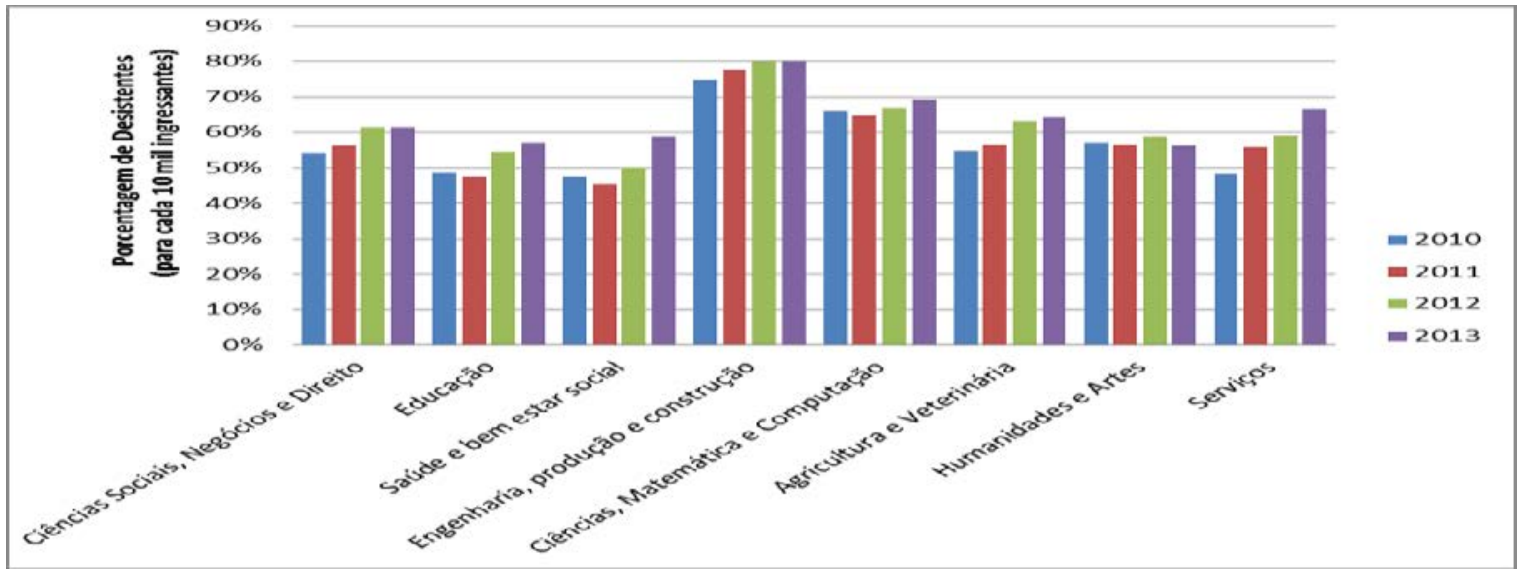

Figura 3. Porcentagem de desistentes por áreas de conhecimento 


\section{Trabalhos Correlatos}

Diante do cenário mundial e nacional, é importante estudar meios para superar a aversão dos jovens pelas áreas de exatas. As subseções a seguir apresentam três exemplos de projetos que visam motivar a candidatura de alunos em carreiras de exatas. A subseção 3.1 trata do programa híbrido de inovação para a diversificação e reforço das capacidades em ciências exatas (ISTEM). A subseção 3.2 aborda o programa de oferecimento de experiências tecnológicas inovadoras para estudantes e professores (ITEST). Na subseção 3.3 é apresentada uma abordagem que utiliza uma gincana para ensino de conceitos computacionais para alunos do ensino médio.

\subsection{ISTEM}

ISTEM é um projeto do Centro de Pesquisa e Aprendizagem de Ciências Exatas (STELAR) dos EUA para desenvolver e determinar estratégias eficazes para envolver alunos no campo das ciências exatas. $\mathrm{O}$ projeto se foca em estudantes da terceira à oitava séries do ensino fundamental, que são atingidos por meio de mentoria nas escolas e experiências externas ao ensino formal. Essa iniciativa recruta, treina e mantém um mínimo de sessenta mentores durante um período de três anos. $\mathrm{O}$ mentor pode ser um aluno de faculdade, um profissional ou um voluntário que realiza duas reuniões por mês com grupos de alunos que são engajados em, no mínimo, cinco experiências informais associadas à ciência. As atividades do programa são baseadas em quatro grandes projetos em engenharia determinados pela Academia Nacional de Engenharia: Energia e Meio Ambiente; Saúde; Segurança e Aprendizagem e Computação. A mentoria busca facilitar a aprendizagem, identificando teorias e métodos relacionados ao cotidiano dos alunos de maneira a ajudá-los a contextualizar os problemas [STELAR 2012].

\subsection{ITEST}

O ITEST é um programa da Fundação Nacional de Ciência da Diretoria para Educação e Recursos Humanos dos EUA que tem como objetivo financiar as melhores práticas para motivar a participação de alunos do ensino fundamental e médio no núcleo de disciplinas de ciência, tecnologia, engenharia e matemática. Essa iniciativa procura implementar e difundir estratégias, ferramentas e modelos inovadores que propiciem o interesse dos alunos por meio de experiências formais e informais, na escola e fora dela. O ITEST financia projetos que divulgam a área de ciências exatas, que orientam os alunos a buscarem trajetórias educacionais apropriadas e que proporcionam $\mathrm{o}$ desenvolvimento de habilidades práticas demandadas pelos empregadores do setor. Experiências que envolvam ativamente a indústria e os negócios ligados à área de exatas são fortemente encorajadas [NSF 2014b].

O ITEST financia, por exemplo, o projeto FUSE Studios, organizado a partir de uma sequência de desafios envolvendo atividades práticas. Esses desafios são orientados pelo interesse de grupos de alunos que são apoiados por uma tutoria on-line de especialistas do setor. A competição envolve o julgamento dos artefatos produzidos pelos alunos que são premiados com base nas realizações coletivas em cada desafio. $\mathrm{O}$ projeto pretende investigar como a temática dos desafios afeta o interesse de jovens [NSF 2014a]. 


\subsection{Aplicação de gincanas no ensino da computação}

Barbosa et al (2015) propuseram uma gincana a fim de levar conceitos da computação como, por exemplo, habilidades relacionadas ao raciocínio lógico e à resolução de problemas, para alunos de $1^{\circ}$ ano do ensino médio. A gincana ocorreu em duas etapas, com três encontros presenciais semanais. Em cada encontro, monitores distribuíram os participantes em grupos de, em média, seis alunos, para os quais um tópico da computação necessário para aprendizagem da programação foi apresentado. Inicialmente era realizada uma explicação conceitual através de artefatos familiares aos participantes e, em seguida, os alunos resolviam uma atividade relacionada com o conceito aprendido. Segundo os autores, o objetivo de ensinar conceitos computacionais foi atingido satisfatoriamente pelo estímulo aos alunos para raciocinar e desenvolver sua própria estratégia de resolução de problemas.

\section{A proposta do DJE}

O Desafio Jovem Engenheiro (DJE) é um torneio criado para incentivar alunos do ensino médio a se candidatarem a cursos superiores na área de exatas e tecnológicas. $\mathrm{O}$ DJE se utiliza de projetos desafiadores direcionados a equipes que, dependendo de seu desempenho, podem ser premiadas na etapa final. As equipes são compostas por até quatro integrantes, sendo quatro estudantes do ensino médio, ou ainda, três estudantes e um mentor que auxilia a equipe na resolução do projeto. O mentor pode ser professor de uma disciplina de exatas do ensino médio, um professor universitário ou um aluno de graduação em exatas.

O DJE é estruturado em duas etapas classificatórias e uma etapa final. As etapas classificatórias selecionam as equipes com as melhores pontuações para continuar no torneio na etapa subsequente. A primeira etapa possui três projetos desafiadores, enquanto a segunda conta com dois. As dez equipes melhores colocadas ao final das etapas classificatórias são selecionadas para competirem na etapa final. O tema de cada projeto é um problema colocado em um determinado contexto, no qual os alunos precisam explorar habilidades de uma área das exatas para encontrar a solução. Os projetos desafiadores são propostos por professores universitários ou por empresas patrocinadoras do torneio [DJE 2014].

$77 \%$ dos adolescentes brasileiros são usuários da internet [CGI.BR 2014]. Por este motivo, a Internet é utilizada como principal ferramenta de Tecnologia Digital de Informação e Comunicação (TDIC) para realização do DJE, tornando possível a participação de estudantes de diferentes perfis e residentes em todos os estados do Brasil. Como o DJE ocorre totalmente online, é necessário o uso de uma ferramenta que simplifique a comunicação entre os participantes e a organização. Para isso é utilizada a ferramenta TelEduc. As equipes recebem o projeto através do Teleduc e é estabelecido um prazo para que resolvam o problema e enviem a solução.

\section{Materiais e métodos}

A falta de profissionais de tecnologia no Brasil implica a necessidade de iniciativas como o DJE, que visa à atração de jovens para as carreiras de exatas. Os trabalhos correlatos - especialmente o ITEST — oferecem exemplos da utilização de projetos desafiadores apoiados por mentoria e ferramentas online para motivar equipes de alunos 
e desenvolver suas habilidades. Nesse sentido, a implementação do DJE se deu a partir da utilização dos materiais e métodos que são descritos a seguir. A Subseção 5.1 trata da aprendizagem baseada em projetos. As subseções 5.2 e 5.3 apresentam as ferramentas de TDIC utilizadas pelo DJE: o TelEduc e as redes sociais, respectivamente.

\subsection{Aprendizagem baseada em projetos}

A Aprendizagem Baseada em Projetos (PBL) é destinada a reforçar as capacidades dos alunos em projetos, trabalho e comunicação interpessoal através de experiências desenvolvidas na solução de problemas como uma equipe. PBL tornou-se muito utilizada no ensino da engenharia, pois a indústria requer graduados com habilidades voltadas para a execução de projetos [HAN, CAPRARO e CAPRARO 2014, YUKAWA et. al 2012]. Nessa abordagem, os estudantes utilizam etapas de planejamento e execução de um projeto para solucionar um problema. A aprendizagem autodirigida ocorre quando a equipe se une em um processo que envolve procurar, conciliar e selecionar as soluções para resolver o problema [HONG et. al 2012].

PBL busca capturar o interesse dos alunos através de uma situação do mundo real e provocar reflexões a respeito de como eles adquirem e aplicam o novo conhecimento em um contexto de resolução de problemas. O papel do professor é de facilitar a resolução, formulando perguntas que auxiliem os alunos a encontrarem a solução. Uma das vantagens dessa abordagem é que pode ser aplicada dentro e fora da sala de aula, uma vez que o mentor, ao contrário de um instrutor, deve avaliar principalmente como os estudantes aprenderam com essa experiência [EFSTRATIA 2014].

\subsection{TelEduc (EaD)}

A comunicação entre os organizadores do DJE e as equipes é feita através do TelEduc, uma ferramenta de Ensino a Distância $(\mathrm{EaD})$ desenvolvida pelo Núcleo de Informática Aplicada à Educação (NIED) da Universidade Estadual de Campinas (UNICAMP). Essa ferramenta integra todo o torneio em um único ambiente e permite a publicação dos enunciados dos projetos. Isso facilita a comunicação entre as equipes e os organizadores que podem interagir através do correio eletrônico. Além disso, o TelEduc dispõe de portfólios para as equipes publicarem as resoluções de cada projeto [NIED 2016]. Uma característica importante do TelEduc, além de suas inúmeras funcionalidades, é o fato de ser uma ferramenta livre com código aberto [TELEDUC 2016].

\subsection{Redes Sociais}

Sackey, Nguyen e Grabill (2015) apontam a necessidade de utilização de duas plataformas, usadas de formas distintas, a fim de permitir o engajamento dos participantes. No TelEduc, a interação é restrita apenas ao conteúdo do DJE e ocorre somente quando o aluno está conectado à plataforma. Por outro lado, as redes sociais permitem um envolvimento com o participante para além do momento da entrega dos projetos, através da publicação de curiosidades e notícias, em uma plataforma que ele utiliza frequentemente. Estudos relatam que cerca de 90\% dos adolescentes entre 15 e 17 anos possuem um perfil nas redes sociais [CGI.BR 2014]. A opção por um veículo mais familiar ao cotidiano do jovem tem o intuito de dar maior visibilidade e divulgar informações pertinentes ao torneio. O DJE utiliza uma página do Facebook para 
publicar informações e esclarecimentos sobre os projetos, o período de inscrição, além de diversas notícias e curiosidades sobre a área de tecnologia. O Facebook se apresenta como importante ferramenta para auxiliar a atração de participantes para o torneio.

\section{Resultados e discussão}

O DJE ocorre anualmente desde 2013. Em duas edições, reuniu aproximadamente 180 equipes oriundas de diversas regiões do Brasil. A primeira edição teve caráter experimental no que diz respeito à implantação da proposta. Apesar disso, o número de equipes inscritas (90 equipes) e a repercussão positiva motivaram a realização de uma nova edição. No ano de 2014 ocorreu a segunda edição do DJE, que contou com a participação de 326 estudantes distribuídos em 89 equipes.

A Figura 4 apresenta informações referentes ao total de equipes inscritas na competição de 2014. O gráfico (A) mostra que o envolvimento de indivíduos do sexo feminino é $44 \%$ menor que o de indivíduos do sexo masculino. Esse resultado pode indicar que o interesse de mulheres em cursos de exatas tende a ser menor. O gráfico (B) apresenta a distribuição das equipes inscritas de acordo com as regiões geográficas do país. A região Nordeste teve metade das equipes inscritas, um índice aproximadamente $20 \%$ maior do que o da região Sudeste, segunda colocada em número de participantes. Nessa edição, a região Sul não foi representada, já que não contou com equipes inscritas. O gráfico $(\mathrm{C})$ contém a distribuição dos inscritos pelo tipo de instituição de ensino. Aproximadamente metade dos participantes do DJE é oriunda do setor público de ensino composto por escolas estaduais, escolas técnicas estaduais (ETECs) e institutos federais. O setor privado de ensino respondeu por $32 \%$ do total de inscritos.

A Figura 5 apresenta informações referentes ao total de equipes que se inscreveram, mas deixaram de participar do torneio ao longo das etapas do DJE 2014. As desistências representaram $65 \%$ do total de inscritos. O gráfico (A) mostra que a taxa de desistência de indivíduos do sexo masculino é cerca de $40 \%$ maior do que a de indivíduos do sexo feminino. O gráfico (B) apresenta a distribuição das equipes desistentes de acordo com as regiões geográficas do país. Nesse aspecto, a distribuição das taxas de abandono foi proporcional às taxas de inscrição. A região Nordeste teve metade das equipes que abandonaram a competição, seguida pela região Sudeste com cerca de $30 \%$ de desistentes. O gráfico (C) permite notar que o setor privado de ensino respondeu pelo maior número de equipes desistentes dentre os inscritos no DJE. Das equipes que abandonaram o torneio, aproximadamente $30 \%$ vieram de colégios particulares.

A Figura 6 apresenta informações referentes às dez equipes que obtiveram as melhores colocações nas duas primeiras etapas e, por isso, foram selecionadas para a fase final do DJE 2014. O gráfico (A) mostra que a presença de indivíduos do sexo feminino e masculino foi proporcional às taxas de inscrição. $O$ gráfico $(B)$ apresenta a distribuição das equipes finalistas de acordo com as regiões geográficas do país. Somente equipes do Nordeste e do Sudeste se classificaram para a final do torneio, contando com $60 \%$ e $40 \%$ dos finalistas, respectivamente. O gráfico (C) mostra que, dentre as equipes que se classificaram para a final do DJE, houve apenas representantes de escolas estaduais, ETECs e institutos federais. Estes responderam por $70 \%$ dos participantes melhor classificados. O campeão da segunda edição do DJE foi uma 
equipe do Instituto Federal do Espírito Santo (Sudeste) composta por quatro indivíduos, um do sexo feminino e três do sexo masculino.
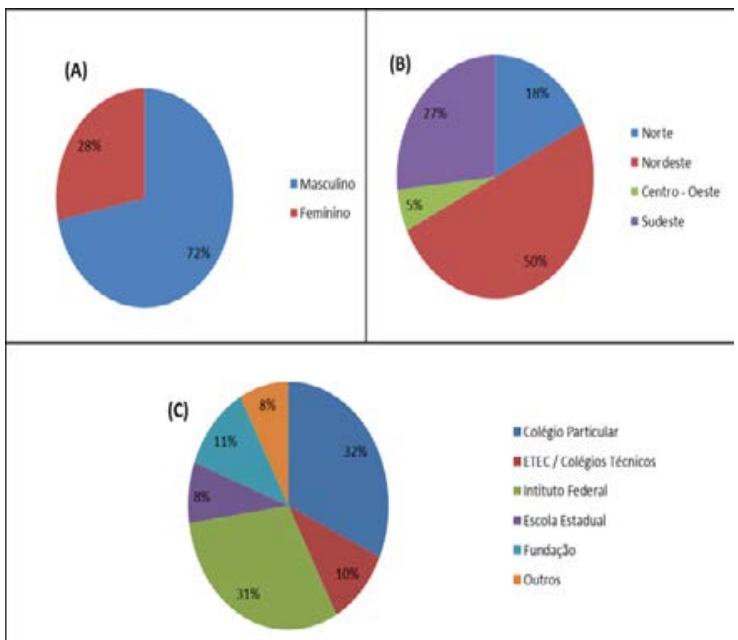

Figura 4. Inscritos edição de 2014.

(A) \% por gênero,

(B) \% de equipes por região,

(C) equipes por tipo de inst. de ensino.

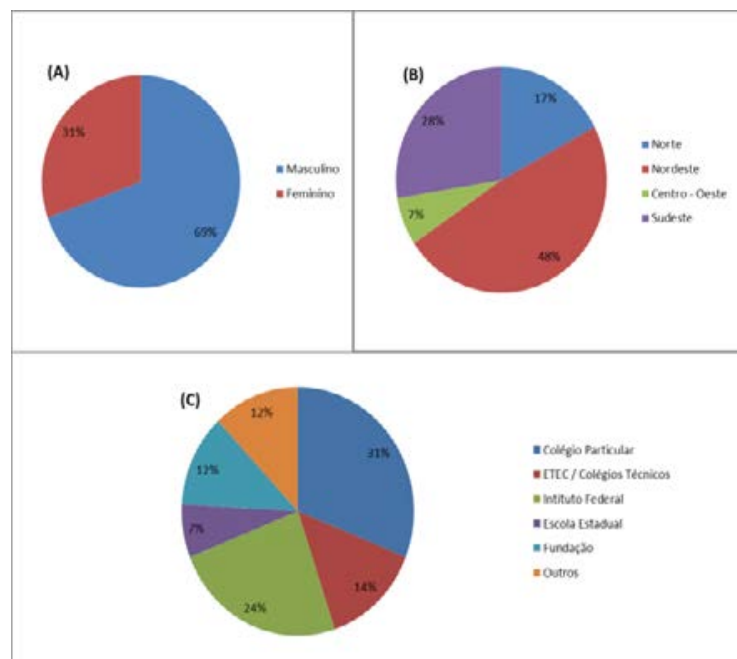

Figura 5. Desistentes edição de 2014. (A) \% por gênero,

(B) \% de equipes desistentes por região, (C) desistentes por tipo de inst. de ensino.

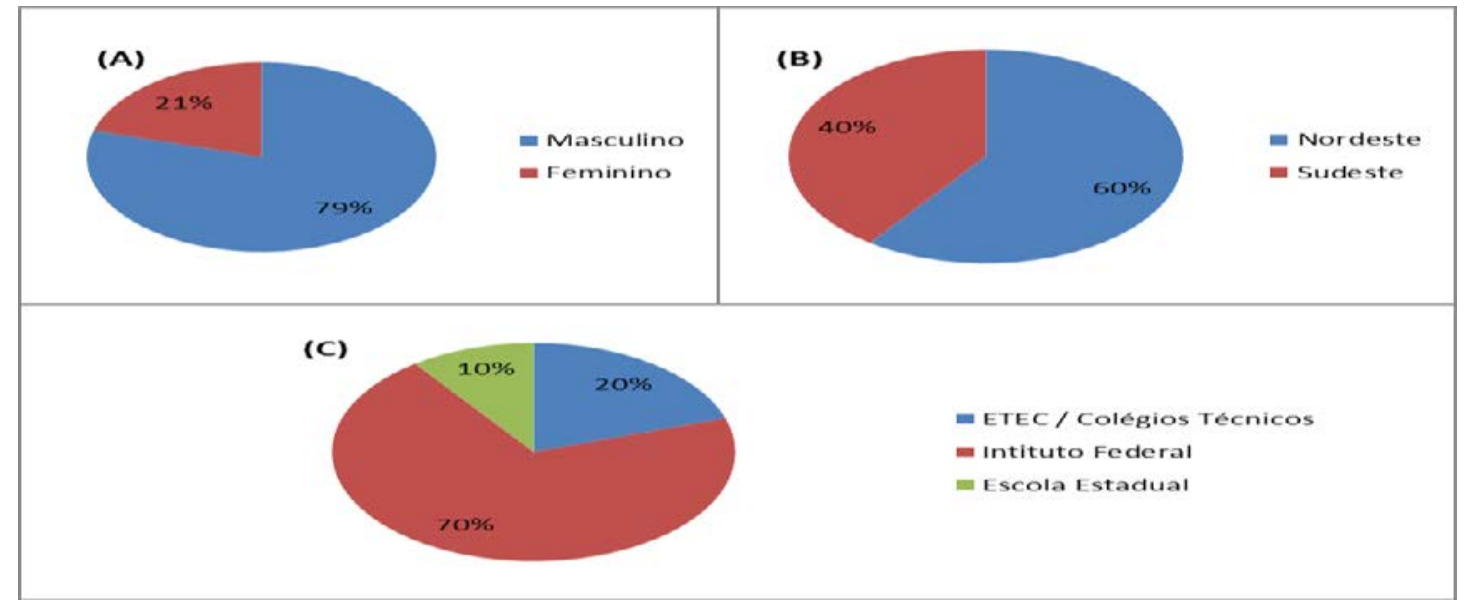

Figura 6. \% finalistas edição de 2014 (A) por gênero, (B) por região, (C) por tipo inst.

\section{Conclusão}

Este trabalho apresenta o Desafio Jovem Engenheiro (DJE), uma proposta alternativa para incentivar jovens a se candidatarem para cursos de tecnologia e engenharia. Essa proposta se estabelece sobre um torneio para alunos de ensino médio, que é composto por projetos desafiadores distribuídos em etapas que visam desenvolver o interesse desses jovens pelas ciências exatas. A competição é inspirada por outras iniciativas semelhantes pelo mundo, entretanto utiliza conceitos de aprendizagem baseada em projetos. Assim, a pontuação de cada etapa depende da elaboração de projetos vinculados à atuação de profissionais da área de exatas, como engenheiros e analistas de sistemas. No ano de 2014, em sua segunda edição, o DJE contou com 326 jovens, que se engajaram, trabalharam em equipe e colaboraram entre si, desenvolveram novas 
habilidades, adquiriram e exercitaram conhecimentos e resolveram problemas a partir de conceitos da área de tecnologia.

A ausência de equipes inscritas da região Sul demanda que nos próximos anos sejam desenvolvidas novas estratégias na divulgação do torneio nos estados do Paraná, Santa Catarina e Rio Grande do Sul. A falta de representantes do Sul do Brasil pode indicar que a utilização das redes sociais (em especial, o Facebook) como ferramenta mobilizadora não surtiu o mesmo nível de efeito nessa região. Também devem ser feitas considerações acerca da baixa participação de indivíduos do sexo feminino. As próximas edições do DJE devem, portanto, preocupar-se com esse público por meio de iniciativas focadas na atração das jovens estudantes para as ciências exatas. Além disso, deve-se investigar a alta taxa de desistência de modo a compreender os motivos que levaram os participantes a abandonar o torneio.

Outra questão a ser abordada trata do impacto do DJE para a escolha da carreira de cada um dos participantes. Para tal, deve-se realizar, futuramente, um acompanhamento dos inscritos de cada edição, a fim de mensurar a real contribuição do torneio para atração e formação dos futuros engenheiros.

\section{Referências}

Barbosa, A., Pereira Neto, A., Oliveira, R., Costa, T., Araújo, A., Costa, F. (2015). O ensino de conceitos computacionais para alunos do ensino médio: relato de experiência de uma gincana e das estratégias utilizadas pelos alunos na resolução das atividades desplugadas. In: Anais do $23^{\circ}$ Workshop sobre Educação em Informática (WEI 2015), Recife, PE.

CGI.BR. (2014). TIC KIDS ONLINE BRASIL 2013: Pesquisa sobre o uso da Internet por crianças no Brasil. Comitê Gestor da Internet no Brasil, São Paulo.

Chen, X. e Soldner, M. (2013). STEM Attrition: College Students' Paths Into and Out of STEM Fields. National Center for Education Statistics, Washington.

CONFEA. (2012). Faltam 150 mil engenheiros no País. Acesso em 04 de 03 de 2016, disponível em CONFEA - Confederação Federal de Engenharia e Agronomia: http://www.confea.org.br/cgi/cgilua.exe/sys/start.htm?infoid=16978\&sid=1206

DJE. (2014). Desafio Jovem Engenheiro - Sobre o desafio. (Faculdade de Tecnologia) Fonte: Laboratorio de Informática, Aprendizagem e Gestão: http://www.ft.unicamp.br/liag/wp/jovemengenheiro/

Efstratia, D. (2014). Experiential education through project based learning. Procedia Social and Behavioral Sciences, 152, $1256-1260$.

Han, S., Capraro, R. e Capraro, M. M. (2014). How science, technology, engineering,and mathematics (STEM) project-based learning (PBL) affects high, middle, and low achievers differently: The impact of student factors on achievement. International Journal of Science and Mathematics Education.

Hong, J.-C., Chen, M.-Y., Wong, A., Hsu, T.-F. e Peng, C.-C. (November de 2012). Developing physics concepts through hands-on problem solving: a perspective on a technological project design. International Journal of Technology and Design Education, 22, 473-487. 
INEP. (2013). Censo da Educação Superior 2013. Instituto Nacional de Estudos e Pesquisas Educacionais Anísio Teixeira, Brasília.

Jin, J. C. e Jin, L. (2014). On the relationship between university education and economic growth: the role of professors' publication. Education Economics, 22, 635651.

Korableva, O. e Litun, V. (2014). The potential of transitive economies' growth based on innovative strategy. WSEAS Transactions on Business and Economics, 11, 725736.

MDIC. (2014). Balança Comercial Brasileira: Dados consolidados. Ministério do Desenvolvimento, Indústria e Comércio Exterior, Brasília.

Meneses, L., Mai, L., Rosario, J., Oliveira, E., Gomes, R. (2015). IntroComp: Atraindo Alunos do Ensino Médio para uma Instigante Experiência com a Programação. In: Anais do $23^{\circ}$ Workshop sobre Educação em Informática (WEI 2015), Recife, PE.

NIED. (s.d.). TelEduc. Acesso em 20 de 02 de 2016, disponível em Núcleo de Informática Aplicada à Educação: http://www.nied.unicamp.br/?q=content/teleduc

NSF. (2014). FUSE Studios: A New, Interest-Driven Model for Engaging Youth In STEM and Career Development Through Challenges and Partnership with Industry. Acesso em 28 de 02 de 2016, disponível em National Science Foundation: http://www.nsf.gov/awardsearch/showAward?AWD_ID=1433724\&HistoricalAward $\mathrm{s}=$ false

NSF. (2014). Innovative Technology Experiences for Students and Teachers (ITEST) . Acesso em 28 de 02 de 2016, disponível em National Science Foundation: https://www.nsf.gov/funding/pgm_summ.jsp?pims_id=5467\&org=DRL

OECD. (s.d.). OECD About. Acesso em 25 de 02 de 2016, disponível em OECD: http://www.oecd.org/about/

Rask, K. (2010). Attrition in STEM fields at a liberal arts college: The importance of grades and pre-collegiate preferences. Economics of Education Review, 29, 892-900.

Sackey, D. J., Nguyen, M. T. e Grabill, J. T. (2015). Constructing learning spaces: What we can learn from studies ofinformal learning online. Computers and Composition, $35,112-124$.

STELAR. (2012). Projects. Acesso em 28 de 02 de 2016, disponível em STEM LEARNING AND RESEARCH CENTER: http://goo.gl/fyzLoy

TELEDUC. (s.d.). Histórico. Acesso em 20 de 02 de 2016, disponível em Teleduc: http://www.teleduc.org.br/?q=historico

Winters, J. V. (2014). STEM graduates, human capital externalities, and wages in the U.S. Regional Science and Urban Economics, 48, 190-198.

Yukawa, T., Iwazaki, T., Ishida, K., Nishigaki, Y., Fukumura, Y., Yamazaki, M. e Miura, H. (2012). Online Collaboration Support Tools for Blended Project-Based Learning on Embedded Software Development — Final Report —. Intelligent Interactive Multimedia: Systems \& Services, 14, 419-428. 